FedUni ResearchOnline

http://researchonline.federation.edu.au

This is the peer-reviewed version of the following article:

Balasubramanian, V., Stranieri, A. (2015) A scalable cloud platform for active healthcare monitoring applications. 2014 IEEE Conference on eLearning, e-Management and e-Services, IC $3 e$ 2014, $10^{\text {th }}-12^{\text {th }}$

December 2014, 93-98.

Which has been published in final form at:

http://doi.org/10.1109/IC3e.2014.7081248

Copyright (C 2014 IEEE. Personal use of this material is permitted. Permission from IEEE must be obtained for all other uses, in any current or future media, including reprinting/republishing this material for advertising or promotional purposes, creating new collective works, for resale or redistribution to servers or lists, or reuse of any copyrighted component of this work in other works. 


\title{
A Scalable Cloud Platform for Active Healthcare Monitoring Applications
}

\author{
Venki Balasubramanian, Andrew Stranieri and Ranjit Kaur \\ Centre for Informatics and Applied Optimisation \\ School of Engineering and Information Technology \\ Federation University, Ballarat, Australia \\ \{v.balasubramanian, a.stranieri, ranjitkaur\}@federation.edu.au
}

\begin{abstract}
Continuous, remote monitoring of patients using wearable sensors can facilitate early detection of many conditions and can help to manage the growing healthcare crisis worldwide. A remote patient monitoring application consists of many emerging services such as wireless wearable sensor configuration, patient registration and authentication, collaborative consultation of doctors, storage and maintenance of electronic health record. The provision of these services requires the development and maintenance of a remote healthcare monitoring application (HMA) that includes a body area wireless sensor network (BASWN) and Health Applications (HA) to detect specific health issues. In addition, the deployment of HMAs for different hospitals is not easily scalable owing to the heterogeneous nature of hardware and software involved. Cloud computing overcomes this aspect by allowing simple and easy maintenance of ICT infrastructure. In this work, we report a real-time-like cloud based architecture known as Assistive Patient monitoring cloud Platform for Active healthcare applications (AppA) using a delegate pattern. The built AppA is highly scalable and capable of spawning new instances based on monitoring requirements from the health care providers, and are aligned with scalable economic models.
\end{abstract}

Keywords- cloud based computing; health condition; early detection, BAWSN

\section{INTRODUCTION}

Analysts have estimated there will be 170 million wearable devices for measuring medical indicators such as ECG, heart rate variability and body temperature, in the world by 2017 [1]. Many of these devices are likely to be operated by hospitals to monitor the progress of discharged patients at home in order to achieve real cost savings in an era of increasing health care costs and reduced government capacity for meet the gap. Some early examples of the use of wearable devices in this way are emerging [2-7].

The use of wearable devices requires the sensor devices themselves and a body area wireless sensor network (BAWSN) so that the sensors can transmit data using a wireless protocol such as Bluetooth or ZigBee to a nearby base station which is often a mobile smartphone. In addition, healthcare applications (HA) are required to process the data in order to raise alarms or perform other actions. The entire system is known as a Healthcare Medical Application (HMA).
Although hospitals are likely to use and benefit from wearable devices, according to Williams, they will not be able to resource their use [8]. A conservative estimate of the amount of data streamed in to a major city hospital amounts to gigabytes per day [9]. This presents storage problems and processing challenges, which are unlikely to be readily, managed by hospital IT departments.

In addition, we can expect the emergence of a multitude of condition specific applications, each using different subsets of each patient's health data commissioned by diverse healthcare practices. For instance, a rehabilitation clinic may be interested in tracking a patient's gait, while a counselling service may be interested in tracking time domain heart rate variability to detect suicidal depression and a hospital may be interested in real time body temperature and frequency domain heart rate variability for the early detection of sepsis.

An approach for an architecture that can enable hospitals to scale up to accommodate large numbers of wearable devices involves the streaming of data to cloud repositories and the development of HA's that execute in the Cloud as Services. A business model that readily supports this architecture is a pay-per-use model for the Cloud services and repositories [10]. This architecture and business model facilitates convenient, on-demand network access to a shared pool of configurable computing resources (e.g. networks, servers, storage, applications, and services). These can be rapidly provisioned and released with minimal management effort or service provider interaction. A hospital can make use of this business model to host the HAs in the cloud and have service level agreement (SLA) for the usage. A summary of the requirements for a HMA is:

- the facility for diverse HA's managed by different providers to execute on different subsets of the data

- the ability for each HA to start and stop with very limited overhead resources or cost

- the capacity to ensure that each HA accesses only the data it is authorized to access

- the capacity for any health care provider to spawn a new HA with limited overhead or cost

- the capacity to define or ramp up to ensure a high level of security 
- technologies that support business models include 'pay as you go' and other models that ensure sustainability

This paper presents a pilot system using Shimmer sensors streaming to a mobile Tablet device and then to the Australian Nectar research Cloud. Sample applications involve the monitoring of knee rehabilitation following surgery and monitoring the elderly for falls. The remaining of this paper illustrates that the approach has the capacity to realise the requirements. The architecture is presented in Section II before providing implementation details in Section III. The formulation for cost evaluation is given in Section IV, while Section V concludes this paper.

\section{ARCHITECTURE DESIGN FOR APPA}

In HMAs, the health data gathered by the BAWSN from the patient is delivered to the HA. Although the BAWSN achieves the critical function of gathering trustworthy health data from the patient, the HA provides the visualisation of the patients' progress to the doctor and can have many functionalities. Examples include maintaining the electronic medical records in the database up to date, alerting the concerned clinicians about the condition of the patients, the provision of common ground for the patients and care staff to discuss needs in detail and also in private; it can also conceivably have an intelligent algorithm to predict forthcoming emergency situations. The general functionalities mentioned above are under the perspective of the user of this application. However, from the application developers' perspective, the specific implementation of these functionalities differs considerably depending on the health care requirements. For instance, the design of the electronic medical records application differs considerably for patients who are suffering from lymphoma ${ }^{1}$ and heart disease than for those with other functionalities associated with an intelligent algorithm to predict any situation.

To comprehend the above claims in a pragmatic way, one of the objectives in our research was to build a HA to realize the complete end-to-end HMA. And also, in building an HA, it is imperative for our work to have a real-time test-bed to determine how the data generated and gathered from different BAWSNs is used at the other end of the HMA in a real-time environment. Hence, the AppA is built to maintain the electronic medical record and simultaneously monitor two types of patients for medical conditions - those who have undergone knee procedures and recovering at home and elderly residents at home at risk of falling. These two types of patients were selected because:

1. Knee surgery recovery patients typically have rehabilitation plans that involve sequences of exercises that should be stepped up as the knee recovers mobility. The challenge for most patients is to know when their knee has recovered sufficient mobility to step up to the

\footnotetext{
${ }^{1}$ Lymphoma is a cancer in the lymphatic cells of the immune system
}

next rehabilitation level. This is currently performed on advice from an orthopaedist and/or physiotherapist. However, gaining timely access to these specialists is difficult and so rehabilitation is often delayed, leading to problems being detected too late to correct.

2. Observing the physical condition of elderly people or patients in personal environments such as home, office, and restroom has special significance because they might be unassisted in these locations. The elderly have limited physical abilities and are more vulnerable to serious physical damages even with small accidents, e.g. fall. The falls are unpredictable and unavoidable. In case of a fall, early detection and prompt notification to emergency services is essential for quick recovery $[11,12]$.

The two medical conditions above provides a real-time test-bed to explore the concurrent continuous monitoring using AppA as shown in the below Figure 1.

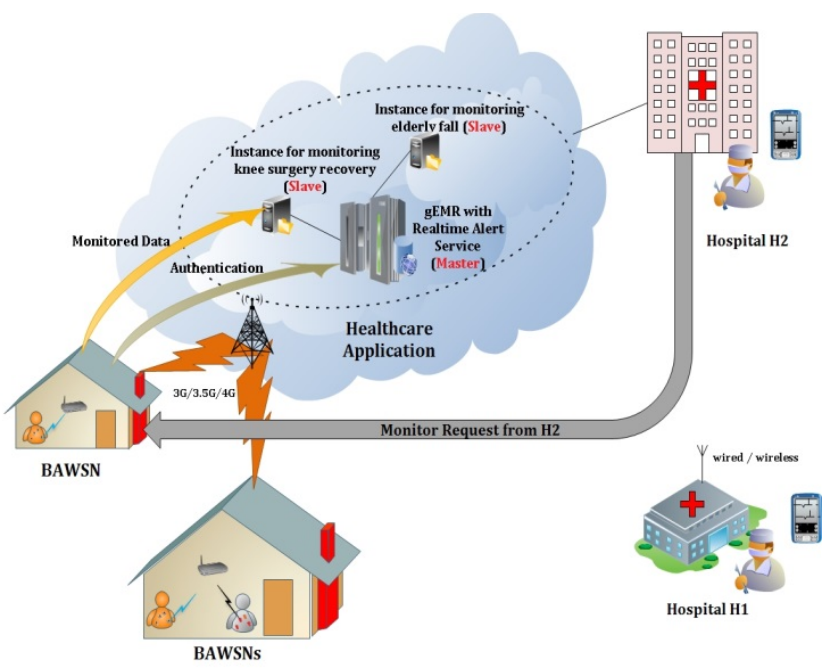

Figure 1: Architecture Design for AppA

Fig. 1 illustrates a generic Electronic Medical Record (gEMR) is devised capable of storing necessary health data for patients. Using the gEMR a master instance is built. The master module can be used as a framework to monitor a variety of medical conditions of the patient. However, in this work two slave instances are spawned to monitor the knee surgery recovery and elderly fall conditions, as shown in Fig. 1 , in the Cloud. Any HA would require health intrinsic data only through BAWSN. The BAWSN uses smartphones to transfer the health data from the body sensors to the slave instance. Therefore, an intelligent mobile application was developed to authenticate the patient as well as to collect the sensors data specifically for those medical conditions.

The architecture design depicts a real-time scenario that might happen when a patient requires continuous monitoring. As shown in Figure 1, the doctors/clinicians can request the patient to start the monitoring process. The 
patient with the wearable sensors attached to their body uses a smartphone application to start the monitor after authenticating their credentials with the master instance. In this way, the hospital ICT can eliminate the onus of authenticating the patient for monitoring. The master instance once authenticates the patient, spawns the slave instance with the required sensor data to be monitored based on the patient conditions and their requirements. The slave instance will be spawned if and only if there is no instance running for a particular hospital for that particular medical condition - this can be identified by using the hospital identification, for example $\mathrm{H} 2$ for Hospital2, as shown in the Figure 1. Please note the patient along with their credentials will enter the hospital identification during the authentication process. This authentication can be scaled up to large numbers of users.

Once spawned, the slave instance will be ready to receive data from the patient's smartphone. The functionalities of the slave instance are to receive the monitored data from the patient and to store the sensor data temporarily. The temporarily stored data can be transferred either to the master instance or directly to the hospital based on the service level agreement (SLA) between the cloud service provider and the hospitals. In addition, doctors from the hospital can login into the slave instance in order to visualize the patient health data and trigger any alert message based on the gravity of the health data.

One of the main aims of the AppA is to reduce the burden for the hospitals in deploying and maintaining the HA with their major functionalities such as patient authentication, continuous health data monitor, health data storage and real-time alert messages. The proposed AppA architecture achieves this by delegating the functionalities among the master and slave instances. The hospital responsibility is to send the request for the patient to start the monitoring and to have a required SLA with the cloud service provider. In the following section we detail the implementation of AppA.

\section{IMPLEMENTATION DETAILS FOR APPA}

The Healthcare Monitoring Application is made up of emerging technologies for various heterogeneous components, therefore, the hardware and software used to build the HMA is also very much heterogeneous. In this section we present in-depth details of the hardware, software and communication infrastructure used in each of the components used in HMA. One of the major components of the HMA is BAWSN, which consists of wearable sensors to monitor the sensor data and the smartphone that acts as a gateway to transfer sensor data to the internet (or to HA). In this work, we used Shimmer sensors [13] and Samsung Tablet as wearable sensors and the gateway respectively.

We selected the followng sensors Accelerometer, Electromyogram (EMG) and Electrocardiogram (ECG) because these variables are used in fall detection algorithms[11-12] and accelerometer data is useful for knee rehabilitation monitoring [14]. The Samsung Tab 2 was used as a gateway to the Australian Nectar Research Cloud (www.nectar.org.au). The following sub-sections present indepth details of the hardware, software and communication infrastructure used in each of the components in HMA.

\section{A. Shimmer Sensors}

To monitor the elderly fall we selected the following sensors Accelerometer, Electromyogram (EMG) and Electrocardiogram (ECG) because these sensors does influence of the detection of elderly fall as given in $[11,14]$. Shimmer is a small sensor platform well suited for wearable applications. The Shimmer3 Bootstrap Loader (Shimmer3 BSL) application allows for the Shimmer unit to be programmed with the appropriate firmware solution when docked in the Shimmer Dock. Shimmer units arrive with BtStream and SDLog firmware. BtStream is a general purpose, fully configurable application to be used with the Shimmer platform. A Shimmer unit programmed with BtStream firmware will stream data via a Bluetooth (BT) connection to a PC, mobile or other Bluetooth-enabled device. The SDLog Firmware is a firmware image which allows logging of data from a Shimmer to the on-board Secure Digital (SD) card. The firmware allows full user configuration of the Shimmer via a configuration file, stored on the SD card. The central element of the platform is the low-power MSP430F5437A microprocessor, which controls the operation of the device with 16Kbyte RAM and 256Kbyte Flash. The CPU has an integrated 16-channel 12bit analogue-to-digital converter (ADC) which is used to capture sensor data. A rechargeable Lithium Polymer battery powers Shimmer sensors. The Shimmer battery is $3.7 \mathrm{~V}, 450 \mathrm{mAh}$ and contains a safety circuit board with over-current protection, which can trigger if a component short is created by a faulty peripheral or if components are bridged while the enclosure is open [13]. The detailed specification of the selected shimmer sensors are given below:

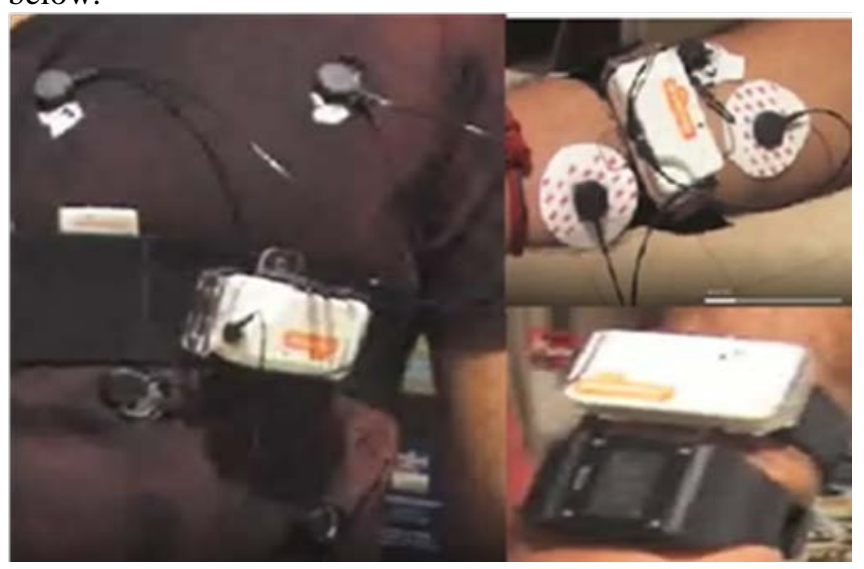

Figure 2. A patient wearing ECG in the chest, EMG in the forearm and a Accelerometer in the wrist. 
1) Accelerometer: By default the shipped Shimmer comes with 3-axis Low Noise Accelerometer and Wide Range Accelerometer array [13]. In addition, the capabilites such as 3-axis Gyroscopes (Angular Rate sensors) and Magnetic Sensor can be enabled as well. However, the Shimmer sensors are capable of sending only one parameter at any given time. In other words, the Set in the sensor hardware is used to send either accelerometer, magnetometer or gyroscope sensor data. By default, the application will sample the 3-axis accelerometer at $51.2 \mathrm{~Hz}$ and send the data over a Bluetooth connection.

2) Electromyogram (EMG): The Shimmer EMG measures and records the electrical activity associated with skeletal muscle contractions and can be used to analyze and measure the biomechanics of human movement. The Shimmer EMG is non-invasive (surface EMG) and therefore the activity it measures is a representation of the activity of the whole muscle or group of muscles whose electrical activity is detectable at the electride site. The Shimmer ECG offers a wireless solution to a host of muscle, gait and posture disturbances in an easy to integrate and ergonomically valuable arrangement [13]. Each EMG board connects to three electrodes, namely, positive, negative and neutral. As shown in Fig. 2, the EMG is attached in the forearm of the patient.

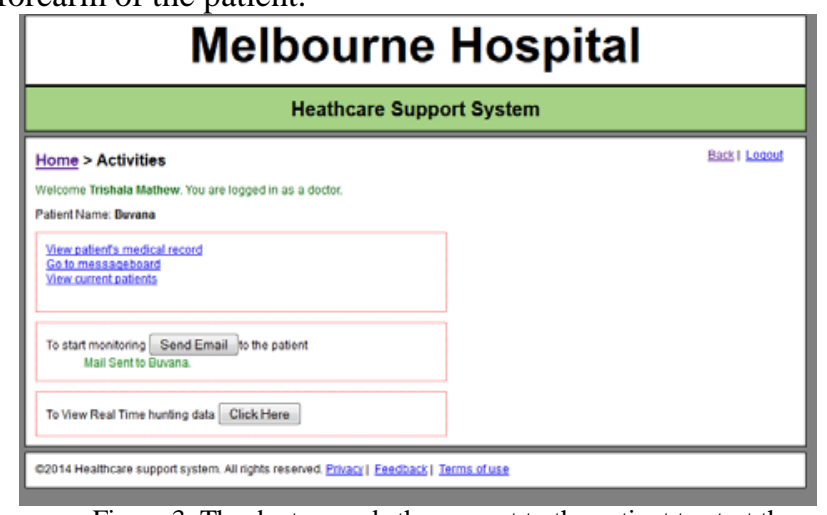

Figure 3. The doctor sends the request to the patient to start the monitoring process

3) Electrocardiogram (ECG): The Shimmer ECG records the pathway of electrical impulses through the heart muscle, and can be recorded on resting and ambulatory subjects or during exercise to provide information on the heart's response to physical exertion [13]. In case of elderly fall detection ECG may give some indication to confirm atrial fibrillation and conduction defects where there is a prolonged PR interval, inferior ischaemia or bundle branch block. The three lead, two channels Shimmer ECG connects to the internal connector pin on the Shimmer main board, and is enclosed within the Shimmer unit, with the application to the skin via four conventional disposable electrodes as show in the Fig.2. In the sub-section below we detail how the sensors data is captured using the smartphone monitoring application.

\section{B. Gateway}

A Samsung Galaxy Tab 2 is used as the gateway in this work. The monitoring application is built using Android SDK [15]. The gateway connects with the selected Shimmer sensors using the Bluetooth. The doctor who wants to monitor the patient sends a request from the hospital website, as shown in the Fig. 3. Once the request from the hospital as shown in the Fig. 4 is received, the patient starts the monitoring application installed in the gateway. The monitoring application prompts the user to enter the creditenals along with hospital identification, as shown in the Fig.5, once authicated by the master instance, the patient would be able to connect the sensors.

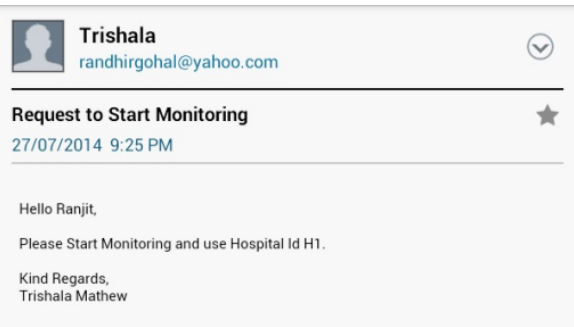

Figure 4. Received request email from the doctor to start the monitoring process

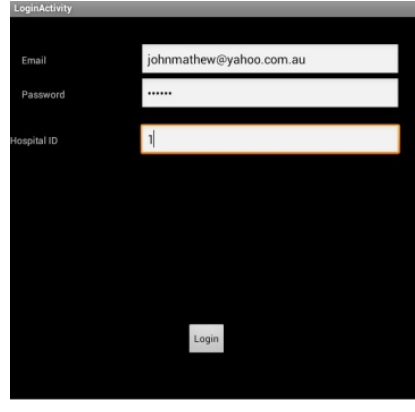

(a)

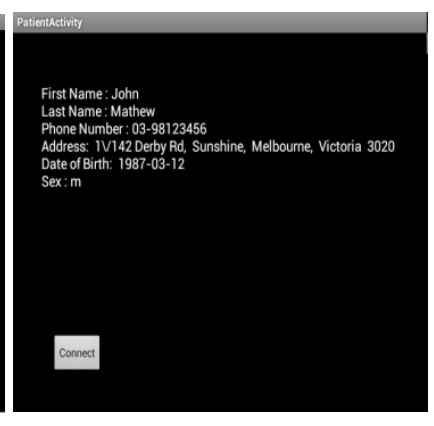

(b)
Figure 5. Login and Authenticated screenshot for the patient in the monitoring application.

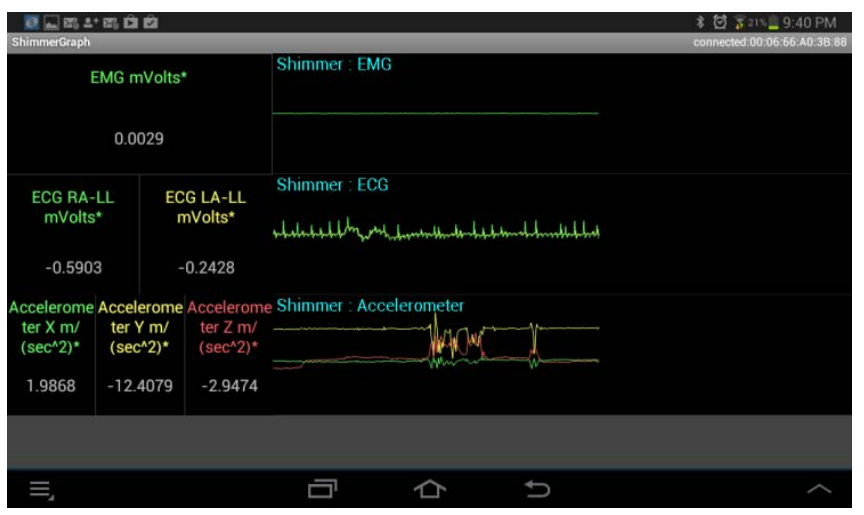

Figure 6. Real-time streaming of sensor data 
The sensors are connected using the connect button and can stream the data of all the three sensors simultaneously as shown in the Fig. 6. The attached EMG in the patient converts input analog skeletal muscle contractions to a digital representation of this signal by assigning a value between 0-4095 to each sample and this will be output from the shimmer EMG module. Whereas, the attached ECG in the patients converts the input analogue signal, from four leads right arm (RA), left arm (LA), left leg (LL) and right leg (RL), in channel 1 and 2 to a digital representation of this signal by assigning a value between 0-4095 to each sample. The accelerometer gives the current position of the subject as $\mathrm{x}, \mathrm{y}$ and $\mathrm{z}$ coordinates. The sensed data from the monitoring application is sent directly to the slave instance spawned by the master instance. Details of the Cloud instances in AppA are presented in the following section.

\section{Cloud Instances}

The slave and master instances are shown in Fig. 7 and Fig. 8 respectively. The slave instance not only enables the doctors to visualize the condition of the patient in real-time but also allows the doctor to send alert messages manually to the patient in case of any abnormalities.

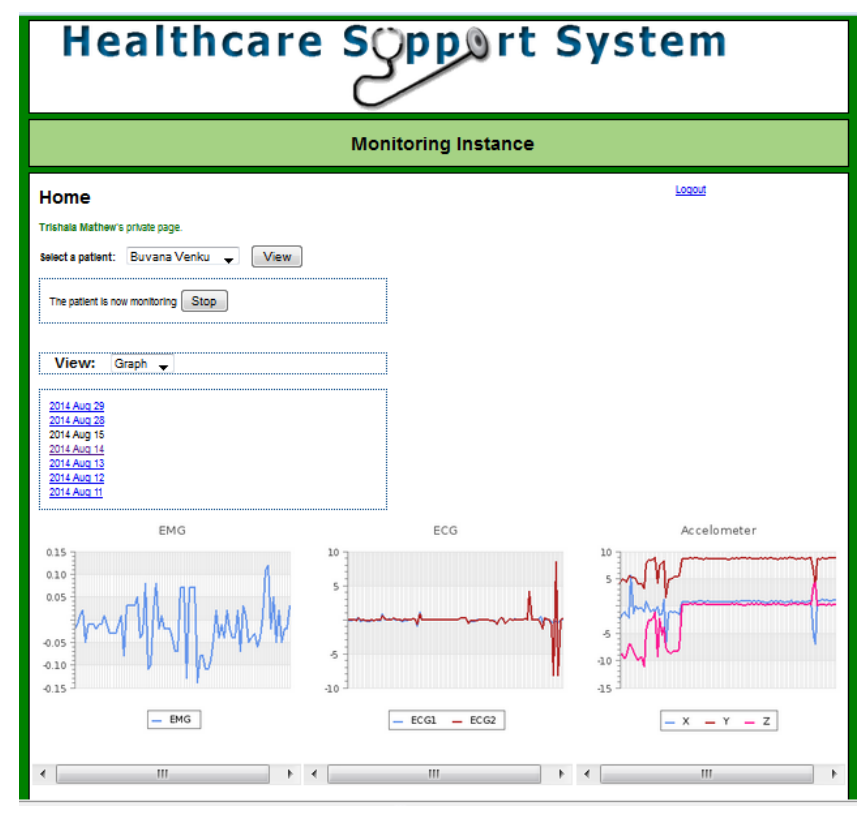

Figure 7. Slave instance shows the EMG, ECG and Accelerometer graph for a patient

The patients are also given access to their data and can visualize it in real-time in the slave instance. In addtion, they can collaborate with doctors using the message board. In this way, the hosiptal can minimise the overhead owing to maintenance of such heavily loaded funcationalities of a HA. The slave instances are spawned dynamically based on the request from the hospital via the patient by using Boto Applicaton programming interface (API) - a python interface to Cloud services [16]. One instance of the slave is spawned per hospital. If the patient from the same hospital makes another request - he/she will be added into the existing running instance.

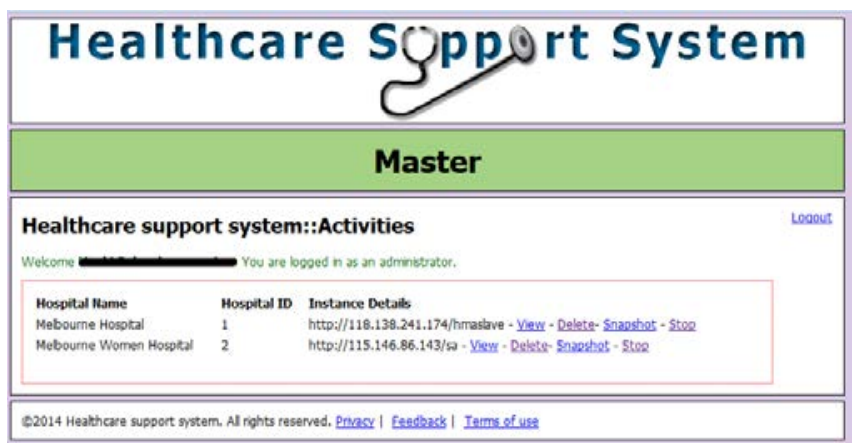

Figure 8. Master Instance shows the active slave instances

The master instance has authentication only for the administrator and have the capacity to view, delete, stop and to take a snapshot of the running slave instance. The master instance shown in the Fig.8 shows the two slave instances running for the hospital capable of monitoring two conditions simultaneously. Currently, the system has been implemented and running in real-time for planned field trials.

\section{FORMULATION FOR COST EVALAUTION}

The AppA is designed in such a way that it can leverage the cloud's pay-per-usage model. The master instance will spawn the slave instance for the hospital only if there is a request from the patient's from the hospital. If there is a slave instance already running for the hospital - any further request from the patient from the same hospital will be added in the existing instance. In this way, the Cloud service provider can charge the hospital only based on the number of hours the instance was running. The formulation below shows the cost estimation for AppA and in-house implementation of healthcare application for hospital $\mathrm{H}$.

Let us consider a hospital $\mathrm{H}$ that has n number of patients to be monitored in real-time for a particulate disease. The total amount of time $\mathrm{T}$ for which the monitoring system was running in order to monitor the $\mathrm{n}$ patients is given by the following equation:

$$
T=\sum_{i=1}^{n} T_{i}
$$

Where, $T_{i}$ is the time duration for monitoring a patient.

It is apparent that the time duration for monitoring $\mathrm{n}$ patients will have overlaps because several patients will be monitored at the same time for $\mathrm{H}$. Therefore, the actual time $T_{A}$ for which the monitoring system would be running is given by: 


$$
T_{A}=\sum_{i=1}^{n} T_{i}-\sum_{i=1}^{n} T_{i} \cap T_{i+1}
$$

The total cost $C$ for running the monitoring system for $\mathrm{H}$ using AppA model is given by the following equation.

$$
C=T_{A} \times C_{H}+C_{R}
$$

Where, $C_{H}$ is expressed in dollars per hour $(\$ / \mathrm{h})$ as we assume $T_{A}$ is expressed in number of hours and $C_{R}$ is the initial registration cost in master instance for $H$. In in-house model, the $\mathrm{H}$ has to incur the following cost for running the same monitoring services.

The capital cost for the hardware components $C_{C}$, maintenance cost for the hardware components $C_{M}$, which includes any on-going upgrades and minimal running cost for the hardware and support $C_{S}$. Please note the hospital $\mathrm{H}$ should have $24 \times 7$ services to replicate the master instance in AppA in order to service the requests from the patient. Therefore, the $\mathrm{H}$ should have proper infrastructure to host the server whose cost is represented by $C_{I}$. Based on the costs involved in running an in-house model, the total cost is given by the following equation:

$$
C=C_{C}+C_{M}+C_{S}+C_{I}
$$

It should be noted that in the above equation, the costs $C_{M}$ and $C_{S}$ are variable and depend on the amount of load the system receives in a given duration of time. Moreover, the hospital $\mathrm{H}$ has to incur the capital cost $C_{C}$ again after certain duration owing to wear and tear of the hardware. Therefore, from equations (3) and (4), it is evident that for in-house model, the $\mathrm{H}$ has to incur overheads in running the monitoring system that has to be investigated further before deployment and also, the in-house model may not be scalable easily.

\section{CONCLUSION}

The AppA approach is aligned with a scalable economic model because it provides the technology for start-up companies to provide HMA services. HMA suppliers can readily be imagined to offer a range of payment models including sourcing the full payment from the client on a per use basis or a combination of per-use and subscription plans from each health care provider and patient. The architecture envisages a plethora of HMA startups ensuring viable competition and also ensuring single vendors do not dominate the market place.

\section{REFERENCES}

[1] Ranck, Judy. “The Wearable Computing Market: A Global Analysis,” GigaOM Pro, July 2012 cited at Electronic Health Reporter at http://electronichealthreporter.com/wearable-devices-wear-well-bein-2014/ Accessed August 2014

[2] Samira H. Habib, Salima Akter, Soma Saha, Fahmida B. Mesbah, Mosaraf Hossain, Liaquat Ali, Cost-effectiveness analysis of medical intervention in patients with early detected of Diabetic Nephropathy in a tertiary care hospital in Bangladesh, Diabetes \& Metabolic Syndrome: Clinical Research \& Reviews, Volume 4, Issue 3, JulySeptember 2010, Pages 123-127

[3] L. Blair, J. Harrison, D. Pahal, C. Clark, J. Allen, Sensing Fluid: The Use of Remote Monitoring Early Detection of Heart Failure to Avert Hospital Admission, Canadian Journal of Cardiology, Volume 29, Issue 10, Supplement, October 2013, Page S395,

[4] R. Agrawal and H.R. Arntz, "Sudden cardiac death does not always happen without warning" In: American Heart Association journal report (2006), pp. 93-98.

[5] Chen, W.L., Chen, J.H., Huang C.C., Kuo, C.D., Huang, C.I., and Lee, L.S. 2008. Heart rate variability measures as predictors of inhouse mortality in ED patients with sepsis. American Journal of Emergency Medicine (2008) 26, 395-401.

[6] E. Ergezen, R.W. Hart, R. Philip, and R.M. Lec, "A high frequency thickness shear mode (TSM) sensor for detection of biomarkers for prostate cancer," Frequency Control Symposium, 2008 IEEE International, 19-21 May. 2008, pp. 341-345.

[7] Ping Huang, Chao-Ying Huang, Shen-Ing Liu, Hybrid intelligent methods for arrhythmia detection and geriatric depression diagnosis, Applied Soft Computing, Volume 14, Part A, January 2014, Pages 38-46, ISSN 1568-4946.

[8] J. Williams Wearable Devices Tell Patient Health Stories-but Hospitals Aren't Ready for Them in Health Care Business News Aug 72014 http://www.hfma.org/Content.aspx?id=24023

[9] State of Victoria 2014. Royal Melbourenn Hospital Performance Report. http://performance.health.vic.gov.au/Home/Report.aspx?ReportKey= 7\&HospitalKey=79 Accessed 12 Aug 2014

[10] Nabil Sultan, Making use of cloud computing for healthcare provision: Opportunities and challenges, International Journal of Information Management, Volume 34, Issue 2, April 2014, Pages 177-184

[11] Woon-Sung Baek; Dong-Min Kim; Bashir, F.; Jae-young Pyun, "Real life applicable fall detection system based on wireless body area network," Consumer Communications and Networking Conference (CCNC), 2013 IEEE , vol., no., pp.62,67, 11-14 Jan. 2013.

[12] Prevention of falls in the elderly: http://www.patient.co.uk/doctor/prevention-of-falls-in-the-elderly-pro Accessed 12 July 2014.

[13] Shimmer sensor : http://www.shimmersensing.com/ Accesssed24 January 2014.

[14] A. T. van Halteren, R. G. A. Bults, K. E. Wac, D. Konstan- tas, I. A. Widya, N. T. Dokovski, G. T. Koprinkov, V. M. Jones, and R. Herzog, "Mobile patient monitoring: The mobihealth system," The Journal on Information Technology in Healthcare, vol. 2, no. 5, pp. 365-373, October. 2004

[15] Android SDK http://developer.android.com/sdk/index.html, Accessed 24 January 2014

[16] Boto https://boto.readthedocs.org/en/latest/, Accessed April $8^{\text {th }} 2014$. 